\title{
Research on Virtual Reality 3D Exhibition Hall based on the Smart Campus of Geely University
}

\author{
Yuxian Bai*, Junjie Fan, Xuan Luo, Qingbo Liu \\ College of Art and Design, Geely University, Chengdu 641423, Sichuan Province, China \\ *Corresponding author: Yuxian Bai, baiyuxian@bgu.edu.cn
}

\begin{abstract}
With the support of Geely college, this study aims to solve the problem that teachers and students' works cannot be displayed under special circumstances. Using this set of applications breaks through many conditions such as time and region, shows learning style to teachers and students and the outside world, and provides a way for knowledge dissemination and sharing.
\end{abstract}

Keywords: UE4; 3D exhibition hall; Blueprint

Publication date: November 2021; Online publication: November 30, 2021

\section{Introduction}

Art and engineering class closing exhibition and graduation work exhibition in colleges and universities have always been an important form of teaching in colleges and universities, but the sudden epidemic makes the work exhibition under the front line unable to be held normally. Then online exhibition becomes an effective solution. As an advanced technical means, virtual exhibition hall greatly reduces the consumption of resources and energy, weakens the dependence on region and capital, truly realizes the never-ending exhibition hall platform, and permanently retains the excellent works of teachers and students and data access. This technology is not limited to professionals, as long as it can be displayed or the contents of the exhibition can use this technology. The application breaks through the restrictions of time, place and platform, and can use mobile phones, PCs, VR and other devices to show more people the style of teachers and students. Promote the dissemination of culture and education and make up for regional restrictions.

Through the research of this subject, the problem that the works of teachers and students cannot be displayed under special circumstances is solved, so that the exhibition hall breaks through many conditions such as time and region, can display to more teachers and students and the outside world, and speed up the dissemination, exchange and sharing of knowledge through network means. Moreover, the platform has the characteristics of autonomy, can be designed and adjusted flexibly, and is not limited by the third party.

\section{Development tool}

In the model part involved in the application, SketchUp, 3dmax and Maya are used to complete the design and production of venues and models, Photoshop is used to complete the design and production of maps, import the three-dimensional model to UE4, complete the production of light, PBR material ${ }^{[1]}$ and dynamic simulation in UE4, and write interactive scripts through visual $\mathrm{C}++$ and blueprint ${ }^{[2]}$ to simulate the first person vision. The system structure is shown in Figure 1. 


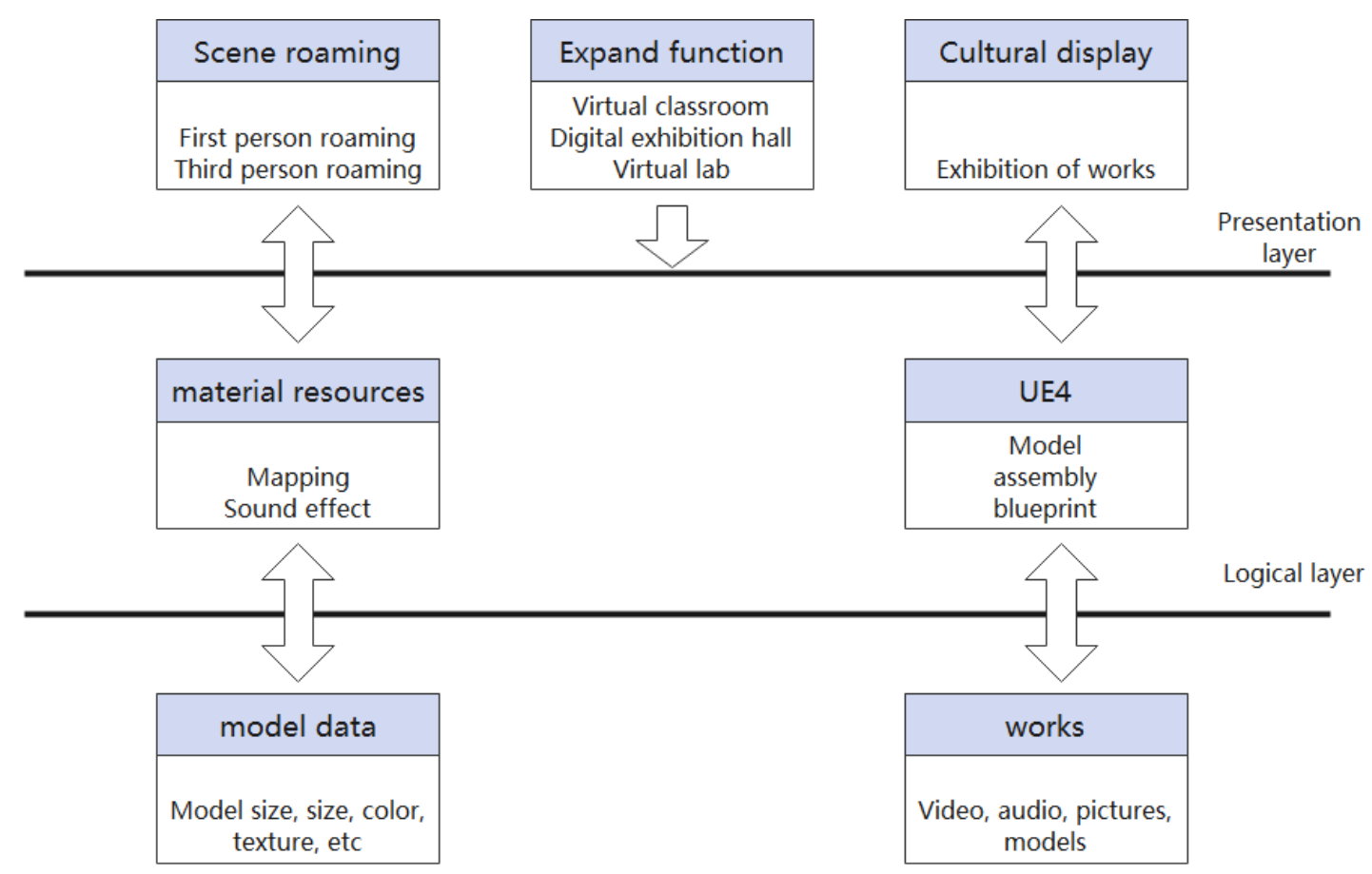

Data layer

Figure 1. The system structures

\section{Material preparation collection}

At the beginning of the production, we collected a lot of information about the three-dimensional virtual exhibition hall, compared the current offline and online virtual three-dimensional exhibition halls, and finally determined the exhibition form and exhibit type of each part of the exhibition hall. Since the school is in the construction stage and there is no physical exhibition hall, we designed the three-dimensional exhibition hall ourselves. The types of exhibits include sculpture, plane products, environmental art, automobile, etc.

\section{Model processing and import}

Due to certain requirements on the number of faces and specifications of the model, Therefore, virtual geometry technology ${ }^{[3]}$ is used all basic 3D models are made by Maya and 3DMAX, and then imported into UE4 engine by Max or Maya through the plug-in officially provided by UE4. The high modulus part is made in UE4, and the map is only given basic materials in Maya and 3DMAX. After importing UE4, use the BPR material editor and re edit all materials based on the HD materials officially provided by UE4.

\section{Lights and materials}

As the scene is a closed design, in order to simulate the real light as much as possible, the scene light production adopts the way of light array. Considering the high requirements of GPU light on computer, the CPU mode is used to build the light in the whole scene.

The models of the whole three-dimensional exhibition hall have many different categories, so it takes a long time to make the materials. The paint materials of cars are designed again based on the paint material effect on the official website. The materials of other parts are designed by ourselves.

\section{Blueprint interaction}

The interactive script of the scene is encapsulated in the function for easy calling. This design uses the first 
persons' perspective, which can give a good sense of substitution. The car interaction part is designed with light switch, paint switch and independent UI control panel. When browsing the scene, you can call out the UI panel according to the needs of "tourists."

The product part is displayed in a dynamic way. When the "tourist" approaches the product, the product introduction and description are dynamically realized in the form of HUD above the booth facing the "tourist." After leaving the booth, the HUD automatically disappears.

The animated character works in the exhibition hall are displayed in a dynamic trigger way. When the "visitors" approaches the animated character, the character makes some specific actions, and the action stops after leaving the booth. This makes the animated character "come alive" and more visually expressive and infectious. A media player is added to the in the exhibition hall, which can be turned on according to the needs of "visitors" to play videos.

\section{Combining content release with smart campus}

Due to the problems of time and hardware equipment, this set of applications is only used on the windows platform. You can provide download links on the school's official website and experience freely after downloading locally, or set up a three-dimensional exhibition hall server in the future to provide client download for network experience, and constantly update and iterate for permanent use.

\section{Funding}

Fund Project: general applied project of Geely University in 2021.

Project Name: Research on Virtual Reality 3D exhibition hall based on the smart campus of Geely University.

Project No. JL2021SKC06

\section{Disclosure statement}

The author declares no conflict of interest.

\section{References}

[1] Gu X, Gortler S, Hoppe H, 2002, Geometry Images, Siggraph.

[2] Foley JD, et al., 2013, Computer Graphics - Principles and Practice, 3nd Ed.

[3] Bai YX, Jun FJ, 2021, The Influence of Virtual Geometry Technology on Animation Industry, Learning \& Education 10(2): 92-93. 